\title{
Quantitative Analysis of Gene Expression During Calcium Homeostasis in $E$. coli
}

\author{
Muhammad Arif ${ }^{1}$ and Louis S Tisa ${ }^{1 *}$ \\ ${ }^{I}$ Department of Molecular, Cellular and Biomedical Sciences, University of New Hampshire, Durham, NH, USA.
}

\begin{abstract}
Despite a wealth of knowledge regarding calcium's role as secondary messenger in eukaryotic cells, relatively little is known about calcium homeostasis in bacteria. Our study employing molecular genetics and transcriptome analysis of calcium homeostasis previously exhibited a calcium regulation of gene expression in elevated or depleted calcium throughout $E$. coli genome. Here we report the results of our subsequent investigation into quantitative analysis using quantitative reverse transcriptase PCR (qRT-PCR) of gene expression in subsets of genes from wild-type and mutant strains with addition of calcium and chelation of calcium. Data from qRT-PCR reveal very high upregulation of genes $y j e E$ and $\sec A$ indicating their possible role as overactive calcium efflux systems in $E$. coli. Calcium is highly likely to be involved in stress response as evidenced by upregulation of marB in transposon mutants and by 10-fold induction of rpoS in elevated calcium condition in mutants defective in calcium transport.
\end{abstract}

Key words: calcium, homeostasis, $E$. coli, qRT-PCR.

\section{Introduction}

Calcium ions serve as secondary chemical messengers in eukaryotic cells and tissues including muscle, neural, and cardiovascular tissue. Calcium is also essential for cell cycle regulation, cellular differentiation, and movement ${ }^{1,2}$. Within prokaryotes, calcium participates in spore formation ${ }^{3}$, chemotaxis $^{4,5}$ motility, cell division, and signal transduction ${ }^{2}$. In eukaryotic cells, calcium ions play an important role as a secondary messenger for signal transduction, behavior, and differentiation ${ }^{6}$. The role of calcium ion participation in bacterial behavior and signal transduction has been established by several lines of evidence ${ }^{5,7-11}$.

Similar to eukaryotic cells, Escherichia coli tightly regulates cytosolic free calcium at approximately $100 \mathrm{nM}^{12}$. The mechanism of calcium entry is unknown. Four genes (calA, calC, calD, and chaA) have been identified in E. coli that are involved in calcium homeostasis. In addition, ATP has been proposed to regulate calcium efflux in E. coli through an ATPase ${ }^{13}$.

We previously investigated global gene expression patterns of wild- type $E$. coli under calcium-depleted (addition of $10 \mathrm{mM}$ EGTA) and calcium-elevated (addition of $75 \mathrm{mM} \mathrm{Ca}^{2+}$ ) conditions as compared to cultures grown under unstressed conditions $^{1}$. Diverse genes across the genome were shown to be involved in calcium homeostasis.

For this study, we performed a comparative analysis of gene expression as quantified by Quantitative reverse transcriptase PCR (qRT-PCR) experiments by focusing on 15 genes that were selected for their purported involvement in calcium homeostasis.

\section{Materials and Methods \\ Strains, plasmids, and phages}

All strains of E. coli used in this study are derivatives of K12 and are listed in Table 1.

Table 1. List of Bacterial Strains

\begin{tabular}{lll}
\hline Strain & Phenotype & Reference or Source \\
\hline CC118 & araD139 & Colin, M and J. \\
& $\triangle($ ara,leu $) 7697$ & Beckwith \\
& $\triangle$ lacX74 phoA $\triangle 20$ & 1985 \\
& galE(galK thi rpsE & \\
& rpoB argEam recAl & Brey and \\
KBT001 & Chemotactically wild & Rosen, 1979 \\
& type purE trp leu proC & \\
& ara lac rpsL metE lysA & \\
& CC118::TnphoA & Arif et al., 2014 \\
Cal134 & CC118::TnphoA & Arif et al., 2014 \\
PhoC23 & Wildtype K12 strain, & Blattner et al., \\
MG1655 & sequenced first. & 1997 ${ }^{17}$ \\
& Tn5 IS50L::phoA & Guttierrez et al, \\
TTnphoA & (KmR) & 1987 ${ }^{18}$ \\
\hline
\end{tabular}

Bacterial Growth Conditions for qRT-PCR Experiments

Overnight cultures were grown in E medium (with appropriate antibiotics) and subcultured the following day in $5 \mathrm{~mL}$ fresh $\mathrm{E}$ medium with an initial OD of 0.05 . Cultures were allowed to 
grow to an OD of 0.2 before appropriate conditions (control, 75 mM Ca or $10 \mathrm{mM} \mathrm{EGTA)} \mathrm{were} \mathrm{added.} \mathrm{The} \mathrm{treated} \mathrm{cultures} \mathrm{were}$ incubated at $37^{\circ} \mathrm{C}$ for approximately 1 hour. Once the cultures reached an OD of 0.4 to 0.5 , pellets were obtained by centrifugation at $16,000 \mathrm{xg}$ for $1 \mathrm{~min}$, frozen immediately in dry ice $/ 95 \% \mathrm{EtOH}$ and stored at $-80^{\circ} \mathrm{C}$ freezer.

\section{RNA extraction}

RNA was isolated using Qiagen RNeasy kit (Qiagen, Inc., Valencia, CA) as per manufacturer's protocol. RNA was eluted from RNeasy columns 2 times with $30 \mu \mathrm{L}$ of RNase-free water. Subsequent to RNA extraction, an aliquot of $10 \mu \mathrm{g}$ RNA for each sample was DNase treated and the DNase was heat-inactivated as per the protocol of New England Biolabs (NEB, Ipswich, MA). RNA concentrations were quantified using a NanoDrop 2000 (NanoDrop Products, Wilmington, DE). The absence of DNA contamination in extracted RNA was confirmed by PCR reaction and subsequent gel electrophoresis.

\section{Reverse Transcriptase Reaction and cDNA Synthesis}

GoScript $^{\mathrm{TM}}$ Reverse Transcription System (Promega Corporation, WI, USA) was used to reverse transcribe RNA templates. Briefly, for every $20 \mu \mathrm{L}$ reaction, approximately $5 \mathrm{mg}$ of RNA and $0.5 \mu \mathrm{g}$ random primers (Promega) were incubated at $70^{\circ} \mathrm{C}$ for 5 minutes, chilled on ice for 5 minutes, and then centrifuged briefly before reverse transcription mix (buffer, $3 \mathrm{mM} \mathrm{MgCl} 2,0.5 \mathrm{mM}$ dNTPs, $1 \mu \mathrm{L}$ reverse transcriptase, water) was added. Annealing at $25^{\circ} \mathrm{C}$ ( $5 \mathrm{~min}$ ), followed by extension at $42^{\circ} \mathrm{C}(1 \mathrm{hr})$, and finally inactivation of reverse transcriptase was carried out at $70^{\circ} \mathrm{C}$ for 15 minutes. The cDNA thus prepared was quantified by NanoDrop 1000 spectrophotometer (Thermo Scientific, Wilmington, Delaware) and diluted to $10 \mathrm{ng} / \mu \mathrm{L}$ working stock in RNAse-free $\mathrm{H}_{2} \mathrm{O}$.

\section{qPCR/Real-Time PCR}

Power SYBR ${ }^{\circledR}$ Green PCR Master Mix (Applied Biosystems, Carlsbad, California) was used for all real-time qPCR reactions to quantify gene expression levels. Each reaction was performed in a total volume of $25 \mu \mathrm{L}$ and contained $5 \mu \mathrm{L}$ of cDNA (50 ng), $12.5 \mu \mathrm{L}$ SYBR Green (final concentration $1 \mathrm{X}$ ), $3.75 \mu \mathrm{L}$ of forward primer (final concentration $120 \mathrm{nM}$ ), and $3.75 \mu \mathrm{L}$ of reverse primer (final concentration $120 \mathrm{nM}$ ). Every sample (housekeeping, controls, unknowns) was represented in triplicate and amplification was performed in a Stratagene Mx3000P QPCR system (Agilent Technologies, Santa Clara, CA). The parameters were as follows: (1) $95^{\circ} \mathrm{C} 10 \mathrm{~min}$, (2) 40 cycles of $95^{\circ} \mathrm{C}$ for $15 \mathrm{~s}$ and $60^{\circ} \mathrm{C}$ for $30 \mathrm{~s}$, (3) $95^{\circ} \mathrm{C}$ for $1 \mathrm{~min}, 55^{\circ} \mathrm{C}$ for $30 \mathrm{~s}$, and $95^{\circ} \mathrm{C}$ for $30 \mathrm{~s}$.

Gene-specific primers were designed using PrimerQuest (Integrated DNA Technologies, Inc., Coralville, Iowa) and are listed in Table 2. The primers were designed to ensure optimum amplification of $100 \mathrm{bp}$ to $150 \mathrm{bp}$ of target gene sequence with a primer $\mathrm{T}_{\mathrm{m}}$ of approximately $60^{\circ} \mathrm{C}$. The specificity of each primer set (used for the amplification of target genes) was verified by analyzing the dissociation curves that were generated with end products of each qRT-PCR reaction. The efficiency of each primer set was determined using standard curves generated by the MxPro software included with the Stratagene qRT-PCR machine (Agilent Technologies, Santa Clara, CA). While the MxPro software automatically set the threshold value for fluorescence, in some cases the threshold values had to be set manually. The threshold cycle $(\mathrm{Ct})$ represented the number of reaction cycles required for fluorescence of SYBR Green (individual reaction) to exceed the threshold value. Relative gene expression (fold change) was analyzed by the Pfaffl method ${ }^{19}$ which allowed for the incorporation of individual primer efficiency values in the calculation of fold change ratios.

The Pfaffl formula employed for relative gene expression studies is represented below:

$\mathrm{R}=\mathrm{E}_{\text {target }} \mathrm{DCt}$ target (calibrator - treated) $/ \mathrm{E}_{- \text {reference }} \mathrm{DCt}$ reference (calibrator
- treated)

Where,

$\mathrm{E}_{\text {-target }}=$ amplification efficiency of the target gene

$\mathrm{E}_{\text {reference }}=$ amplification efficiency of the reference (housekeeping) gene

$\triangle \mathrm{Ct}$ reference (calibrator - treated) $=$ the $\mathrm{C}_{\mathrm{t}}$ of the reference (housekeeping) gene in the calibrator minus the $\mathrm{C}_{\mathrm{t}}$ of the reference (housekeeping) gene in the treated sample.

$\triangle \mathrm{Ct}$ target (calibrator - treated) $=$ the $\mathrm{C}_{\mathrm{t}}$ of the target gene in the calibrator minus the $\mathrm{C}_{\mathrm{t}}$ of the target gene in the treated sample.

\section{Results and Discussion}

\section{Genes of Interest}

Comparative analysis of gene expression as quantified by qRTPCR experiments centered on 15 genes that were selected for their purported involvement in calcium homeostasis based on published data in the recent literature ${ }^{13,20}$ and my preliminary global expression data from Panorama macroarray ${ }^{1}$. Of the 15 genes chosen for this study, 8 genes (cheB, pstS, fts Z, marB, $f a d B, p q i B, y b b O$ and $y f a D$ ) were initially included in the global expression profiling of wild type E. coli MG 1655 grown in control, elevated and depleted calcium conditions during Panorama DNA macroarray experiments ${ }^{1}$. Of the remaining 7 genes, 3 genes $(a t p D$, $\sec A$, and $y j e E)$ were selected based on the calcium efflux observations made by Naseem et al (2009) ${ }^{13}$. The gene atoA was selected due to its implication in calciumregulation of cPHB [complexed poly-(R)-3-hydroxybutyrate] biosynthesis in $E$. coli $^{20}$. The gene $\operatorname{cor} A$ was included because of previously published results indicating that corA mutants were defective in magnesium transport system and were sensitive to calcium ${ }^{16,21}$ also showed that corA mutants were sensitive to $50 \mathrm{mM}$ calcium. The $\operatorname{arcA}$ and $r p o S$ genes were chosen to assess general response to stress in calcium-sensitive mutants. 
Table 2: Primers for qRT-PCR used in the study.

\begin{tabular}{|c|c|c|c|}
\hline Primer & Primer sequence $\left(5^{\prime}-3^{\prime}\right)$ & Gene & Reference \\
\hline $\operatorname{arcA-F}$ & CAGATTCATGGTACGGGACAG & $\operatorname{arcA}$ & This Study \\
\hline $\operatorname{arcA}-\mathrm{R}$ & TGCGTTGATGTTCCTGACTG & $\operatorname{arcA}$ & This Study \\
\hline rpoS-F & AGCTTATGGGACAACTCACG & rpoS & This Study \\
\hline rpoS-R & TCTCAACATACGCAACCTGG & rpoS & This Study \\
\hline rpoB-F & GGTTGGTACTGGTATGGAACG & rpoB & This Study \\
\hline rpoB-R & AGAACGGGTGTATTTGGTCAG & rров & This Study \\
\hline mdh-F & CCATAGACAGGGTTGCAGAC & mdh & This Study \\
\hline mdh-R & TCCTGGCGTTAGTTTTACCG & mdh & This Study \\
\hline ftsZ-F & AGCGAACGATGTACTGAAAGG & $\mathrm{ftsZ}$ & This Study \\
\hline ftsZ-R & GTAGCCCATCTCAGACATTACG & ftsZ & This Study \\
\hline atoA-F & AAGAGCTTCGTGATGGTGAC & ato $\mathrm{A}$ & This Study \\
\hline atoA-R & GCAGAGTGATATGAATACCCTCC & ato $\mathrm{A}$ & This Study \\
\hline atpD-F & TTTTACCTACACCCGCACC & atpD & This Study \\
\hline atpD-R & TGTCAAACTCTCAGGAACTGC & atpD & This Study \\
\hline pstS-F & TTACCATCCAGCACCAGTTC & pstS & This Study \\
\hline pstS-R & CGTTGATTTTGGTGCCTCTG & pstS & This Study \\
\hline marB-F & GCTGCGCTTATTCTCTTTTCC & marB & This Study \\
\hline marB-R & TTATCACTGCCAGTACCCATG & marB & This Study \\
\hline fadB-F & TGTTCGGTTTCTGCCAGTAC & fadB & This Study \\
\hline fadB-R & ATCTCCACAATCCACCCAAC & fadB & This Study \\
\hline aas-F & AGATCCATACTTTGTCGGCAG & aas & This Study \\
\hline aas-R & CAAAACCATCTTCACTTCCCG & aas & This Study \\
\hline cheB-F & TGACTTCTGACCCTTTGCC & cheB & This Study \\
\hline cheB-R & CTGACGCTGGATGTTGAAATG & cheB & This Study \\
\hline ybbO-F & CTGACTTTAATTCCGCTGTGG & ybbO (b0493) & This Study \\
\hline ybbO-R & GGTGAAGGGCGTATTGTGATG & ybbO (b0493) & This Study \\
\hline ycfL-F & GCATCCTCAACGCTTTATAACG & ycfL (b1104) & This Study \\
\hline ycfL-R & GAATGTGCGGGAATGGTAAC & ycfL (b1104) & This Study \\
\hline yfaD-F & GCCGTTGCTGTTTTATCATGG & yfaD (b2244) & This Study \\
\hline yfaD-R & ATCGTCAAACTCATCCAGCC & yfaD (b2244) & This Study \\
\hline corA-F & CGGAACTGGAAGACATCGAAG & corA & This Study \\
\hline corA-R & AGAAGGAGTGAATATGCAGGC & corA & This Study \\
\hline $\sec \mathrm{A}-\mathrm{F}$ & CAAACGAACTGACCAAAGCC & $\sec \mathrm{A}$ & This Study \\
\hline secA-R & CAATATCTGTACCACGACCCG & $\sec \mathrm{A}$ & This Study \\
\hline yjeE-F & CACTTTGATTTGTACCGCCTTG & yjeE (b4168) & This Study \\
\hline yjeE-R & TGTGTATTTCGACATCCGGG & yjeE (b4168) & This Study \\
\hline pqiB-F & GATCGCTGAAAACCGGAAAC & pqiB & This Study \\
\hline pqiB-R & GCTAACGGTCGGGATAATCTG & pqiB & This Study \\
\hline
\end{tabular}

Comparative Analysis of Expression of Genes of Interest in MG1655

For each experimental condition, the expression levels of gene transcripts are shown as a ratio that is relative to expression in the untreated control samples. Some of the common genes included in both Panorama genome array experiments ${ }^{1}$ and qRTPCR experiments (this study) enabled us to compare the expression profiles between the two different approaches used to assess gene expression levels in MG1655 strains under control, calcium-treated and EGTA-treated conditions.
For $c h e B$ gene, a downregulation was observed in the presence of calcium during array ${ }^{1}$ and qRT-PCR analysis (Figure 1), however, array results indicated an upregulation ${ }^{1}$ of the gene under EGTA treatment while qRT-PCR indicated downregulation (Figure 1). As witnessed in qRT-PCR, addition of $75 \mathrm{mM}$ calcium (elevated calcium condition) downregulated cheB almost 10-fold, while treatment with $10 \mathrm{mM}$ EGTA (depleted condition) downregulated $c h e B$ expression 5-fold (Figure 1).

For gene $p s t S$, under calcium addition, qRT-PCR results revealed no change as compared to control condition (Figure 8) and it 
contradicted Panorama genome array results from our previous study which exhibited a two-fold increase ${ }^{1}$. Under calciumdepleted condition, the expression levels were downregulated in both qRT-PCR and Panorama genome array procedures. fts $Z$, $\operatorname{mar} B, \operatorname{fad} B$ and $p q i B$ genes were downregulated under both elevated and depleted calcium conditions in both methods. However, the exact level or fold of relative expression changes may not be identical between the arrays and qRT-PCR procedures.

The fundamental difference between global expression analysis using arrays and individual gene expression measured by qRTPCR procedure is that qRT-PCR places a more individualized quantitative emphasis. As such, array results are usually further verified and validated by qRT-PCR. Our attempts to conduct multiple replicates of global transcriptome profiles of $E$. coli genome were prevented due to resource constraints and limited availability of Panorama E. coli arrays. The results of qRT-PCR experiments therefore were not expected to corroborate all the array results.

Control - MG1655, KBT and CC118

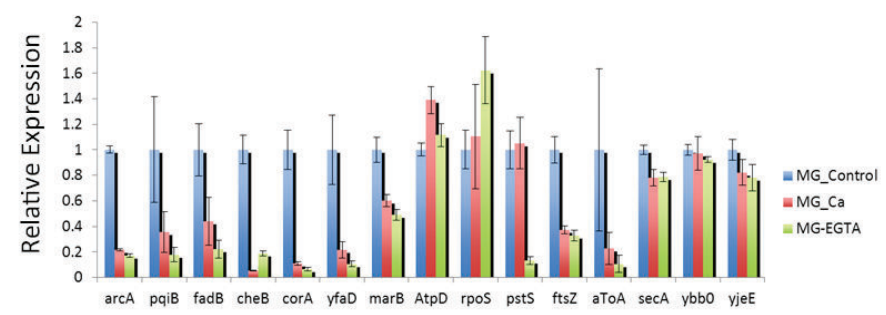

Figure 1. Relative expression of 15 genes under elevated $\mathrm{Ca}^{2+}$ condition (75mM Ca) and depleted $\mathrm{Ca}^{2+}$ condition $(10 \mathrm{mM}$ EGTA) in MG1655. MG1655 (control) was used as the calibrator. The housekeeping gene rpoB was used as the normalizer.

Relative Expression of 3 Parental Wild Type Strains under Control Condition

E. coli MG1655 was used during the Panorama DNA macroarray experiment ${ }^{1}$. To corroborate and validate the preliminary array results, we included strain MG1655 in qRT-PCR experiments concomitant with the investigation of calcium-sensitive mutants and their parental counterparts. Inclusion of MG1655 strain was advantageous because of the following reasons: (i) the readily available sequence information and genome databases, (ii) the preliminary relative expression data obtained from Panorama macroarray $^{1}$, and (iii) the opportunity to look at the big picture which was not just with calcium-sensitive mutants but also to assess expression changes (uprgulation/downregulation) in another wild-type strain during control, elevated and depleted calcium conditions. The relative expression of 15 selected genes in the 3 parental wild type strains in the untreated control condition were very similar to one another (Figure 4).

Because there were no differences in relative expression profiles between the mutants and parents under control condition (Figures 2 and 3), any changes in gene expression under elevated or

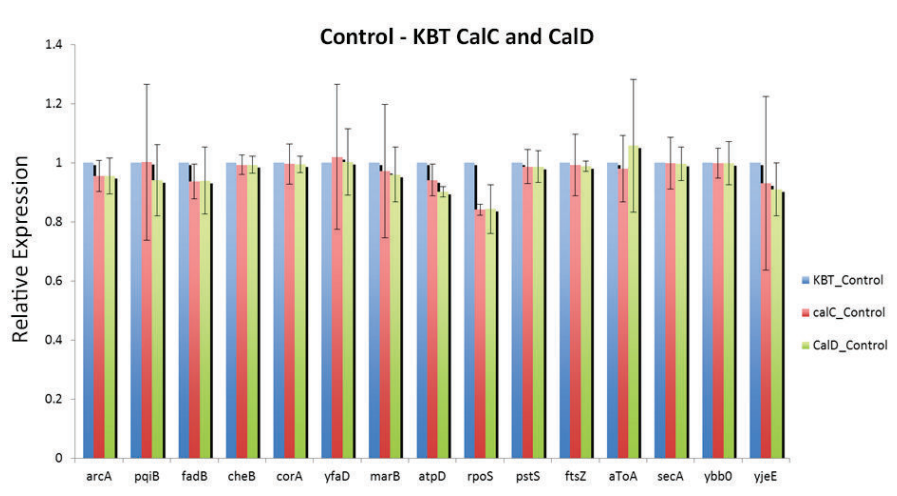

Figure 2. Relative expression of parental (KBT) versus mutant strains (calC and calD). Under control (untreated) condition, no difference was observed in gene expression between calciumsensitive mutants and wild type parent. KBT (untreated) was used as the calibrator. The housekeeping gene rpoB was used as the normalizer.

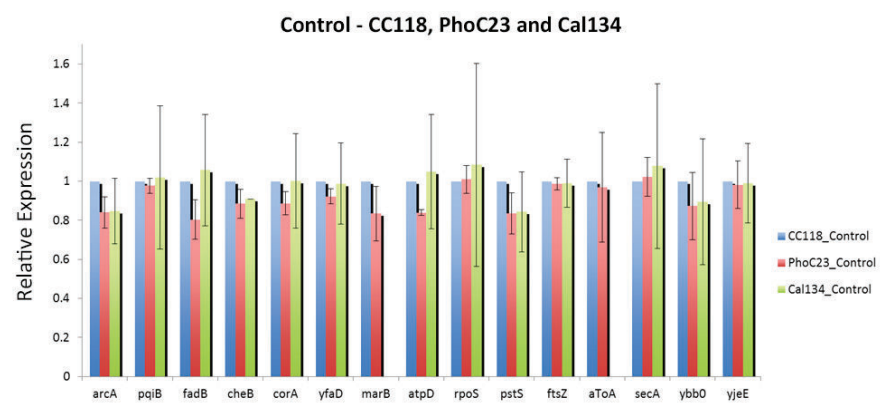

Figure 3. Relative expression of parental (CC118) versus mutant strains (PhoC23 and Call34). Under control (untreated) condition, no difference was observed in gene expression between calcium-sensitive mutants and wild type parent. CC118 (untreated) was used as the calibrator. The housekeeping gene rpoB was used as the normalizer.

depleted calcium conditions would be expected to reflect a genuine response to such conditions. In addition, a similar pattern of relative expression in all wild type strains (MG1655, KBT, CC118) under control conditions as exhibited in Figure 4 would allow us to look at a more comprehensive profile of gene expression (inclusive of results obtained previously from array experiments) during calcium homeostasis.

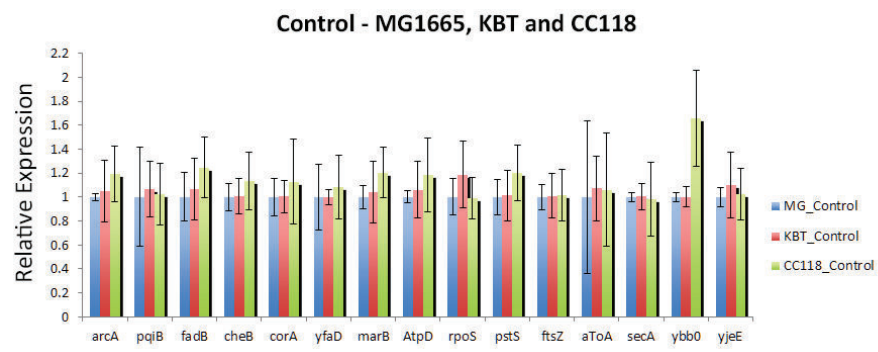

Figure 4. Relative expression of parental wild type strains (MG1655, KBT, CC118). Under control (untreated) condition, no difference was observed in gene expression between MG1655 and the wild type parents. MG1655 (untreated) was used as the calibrator. The housekeeping gene rpoB was used as the normalizer. 


\section{Analysis of Individual Gene Expression}

Due to the fact that the 15 genes selected for the qRT-PCR experiment did not easily classify into functional groups and because there were multiple parent/mutants each with its own control, elevated and depleted calcium conditions, we deemed it more relevant to assess relative expression levels of one gene at a time. Such an effort unambiguously focused on the participatory role of each gene in calcium homeostasis in E. coli.

atpD Expression

Naseem et al (2009) ${ }^{13}$ have recently demonstrated atpD (F1 complex, ${ }^{2}$ subunit of ATP synthase) as a potential calcium efflux gene in $E$. coli. The researchers identified a knockout mutant of $a t p D$ as defective in $\mathrm{Ca}^{2+}$ efflux by measuring cytosolic calcium levels with the aid of calcium-sensitive luminescence of aequorin. The knockout mutant exhibited lowered ATP levels concomitant with defect in calcium efflux. In their experiment, addition of EGTA to atpD knockout cells did not decrease the cytosolic free calcium levels, while EGTA caused a rapid decrease in cytosolic free calcium in wild-type cells.

The CalC and CalD mutants are defective in $\mathrm{Ca}^{2+} / \mathrm{PO}_{4}{ }^{2-}$ symporter activity ${ }^{9}$. While the wild-type parental cells (KBT001) have a cytosolic free-Ca ${ }^{2+}$ level of about $105 \mathrm{nM}$, the free- $\mathrm{Ca}^{2+}$ levels for $\mathrm{CalC}$ and CalD are approximately $1130 \mathrm{nM}$ and 410 $\mathrm{nM}^{7}$. In addition, the mutants are unable to regulate cytoplasmic $\mathrm{Ca}^{2+}$ levels as evidenced by an increase in calcium during addition of calcium to the growth medium and by a decrease in cytoplasmic $\mathrm{Ca}^{2+}$ levels when grown in the presence of EGTA.

In our current study, the qRT-PCR results showed at least a 3-fold upregulation of $a t p D$ in $\mathrm{CalC}$ and CalD mutants during elevated calcium condition (Figure 5). Interestingly, addition of EGTA resulted in a 10-fold upregulation of $a t p D$ (Figure 6) as would be expected if the cells are to efflux out calcium while the chelator in the growth medium drives calcium out of the cell and if $a t p D$ acts as the efflux gene as explained by Naseem et al (2009) ${ }^{13}$. The TnphoA transposon mutant PhoC23 on the other hand exhibited a slight (approx. 2-fold) downregulation of atpD as compared to the parental strain CC118 in both elevated and depleted calcium conditions (Figures 15 and 16). PhoC23 is a tumbly mutant. Whether a defective efflux system is contributing to elevated cytoplasmic free calcium in these mutants is an interesting question - one that would require intracellular calcium measurements. The wild-type MG1655 revealed a minor increase in $a t p D$ expression (approx. 1.2-1.4 fold) in both elevated and depleted calcium conditions (Figure 1). One would presume the wild-type cells to have a functional $a t p D$ regulation to allow for calcium efflux out of the cell as necessary and my results would indicate such a scenario.

\section{marB Expression}

The gene $\operatorname{marB}(219 \mathrm{bp}$ ) encodes a putative protein of unknown function and is thought to have a role in multiple antibiotic resistance and in the regulation of antibiotic efflux pump acr $A B$.

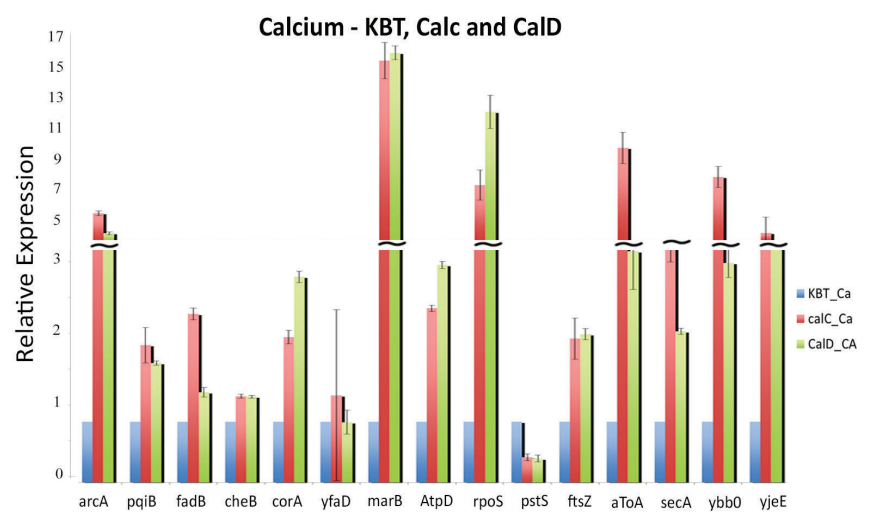

Figure 5. Relative expression of parental (KBT) versus mutant strains (calC and calD) under elevated calcium conditions (75 $m M \mathrm{Ca}^{2+}$ ). KBT (calcium-treated) was used as the calibrator. The housekeeping gene rpoB was used as the normalizer.

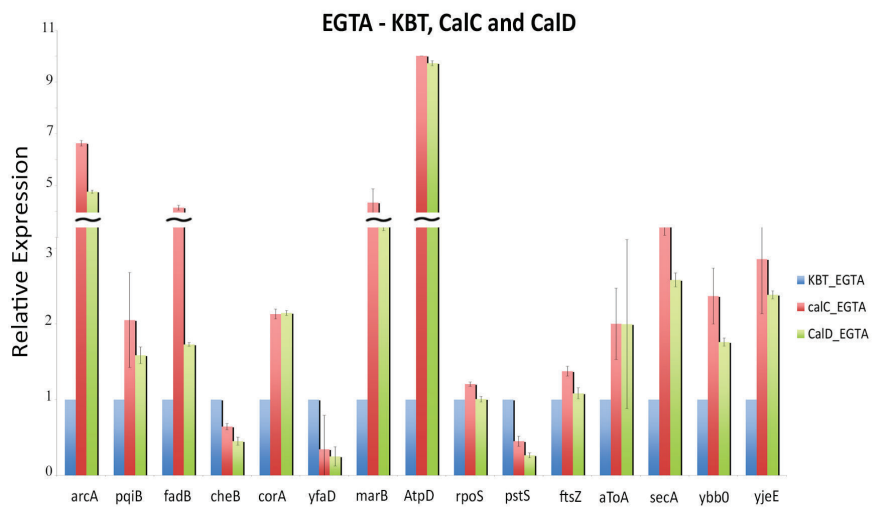

Figure 6. Relative expression of parental (KBT) versus mutant strains (calC and calD) under depleted calcium conditions (10 mM EGTA). KBT (EGTA-treated) was used as the calibrator. The housekeeping gene rpoB was used as the normalizer.

Calcium - CC118, C23 and Cal134

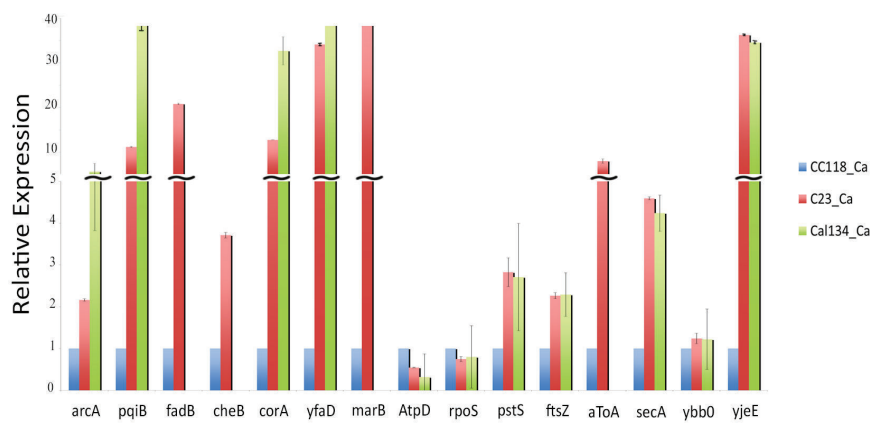

Figure 7. Relative expression of parental (CC118) versus mutant strains (PhoC23 and Cal134) under elevated calcium conditions (75 $\mathrm{mM} \mathrm{Ca}^{2+}$ ). CC118 (calcium-treated) was used as the calibrator. The housekeeping gene rpoB was used as the normalizer.The calcium-sensitive mutant Cal134 failed to yield any $C t$ values in $q R T-P C R$ for fadB, cheB, marB, and ato $A$ genes. 


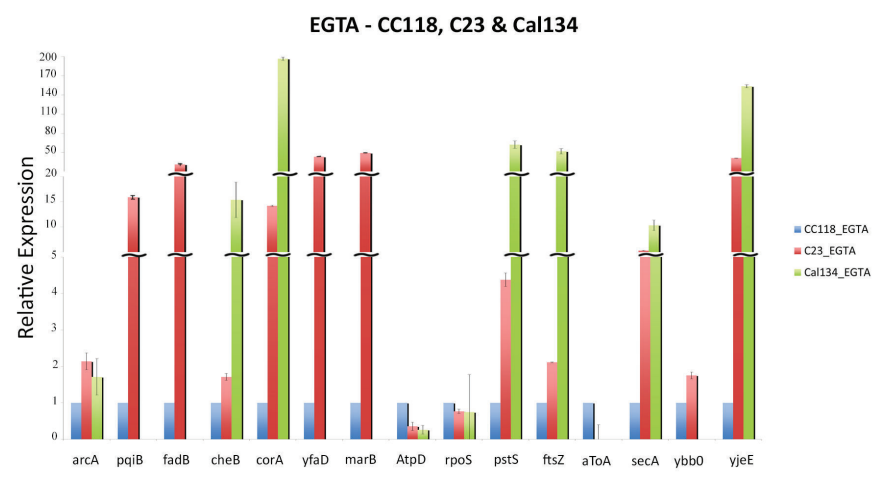

Figure 8. Relative expression of parental (CC118) versus mutant strains (PhoC23 and Cal134) under depleted calcium conditions (10 mM EGTA). CC118 (EGTA-treated) was used as the calibrator. The housekeeping gene rpoB was used as the normalizer.The calcium-sensitive mutant Cal134 failed to yield any $C t$ values in $q R T-P C R$ for pqiB, fadB, yfaD, marB, ato $A$, and ybbO genes.

In the qRT-PCR experiments, the CalC and CalD mutants had 15-fold and 3-fold upregulation of marB under elevated and depleted calcium conditions, respectively (Figures 5 and 6). The transposon mutant PhoC23 exhibited a high upregulation of 40fold in both elevated/depleted calcium conditions (Figures 7 and 8). The wild-type MG1655 strain had a slight downregulation of approx. 2-fold during elevated/depleted calcium conditions as gleaned from qRT-PCR (Figure 1) and were close to the results obtained with Panorama E. coli macroarray experiments ${ }^{1}$.

sec $A$ and yjeE Expression

Bioinformatics analysis of ATP-driven transporters or ATPases in the $E$. coli genome resulted in the identification of two essential genes, $\sec A$ and $y j e E$, that likely code for a $\mathrm{Ca}^{2+}$ efflux pathway ${ }^{13}$. Both of these genes were upregulated (approx. 2-3 fold) in CalC and CalD mutants in the presence of calcium and EGTA(Figures 5 and 6). The elevated calcium conditions resulted in 35-fold upregulation of yjeE in PhoC23 and Cal134 mutants (Figure 7). The yjeE gene was upregulated approx. 40-fold in PhoC23 and 150-fold in Cal134 under depleted calcium (EGTA) conditions (Figure 8). An overactive efflux system may conceivably be involved in these mutants in order to maintain calcium homeostasis.

\section{fadB Expression}

$f a d B$ encodes the \pm subunit of a multienzyme complex that is involved in the b-oxidation/degradation of fatty acids ${ }^{4}$. Mutants $\mathrm{CalC}$ and CalD showed 3-fold higher expression when in elevated calcium, TnphoA mutant PhoC23 exhibited 20-fold upregulation in calcium and 30-fold upregulation when grown in the presence of EGTA (Figures 7 and 8).

\section{pst $S$, arc $A$, and rpoS Expression}

The periplasmic phosphate binding protein/phosphate transporter gene pst $S$ expression was downregulated 2-fold in both elevated/ depleted calcium conditions for both CalC and CalD mutants (Figure 5 and 6). However, with depleted calcium condition, pstS expression was upregulated 5-fold in PhoC23 and 60-fold in Cal134 (Figure 8). The wild-type MG1655 cells showed a downregulation of 10-fold in depleted calcium condition (Figure 1).

The magnitude of upregulation (60-fold) in the case of Cal134 during depleted calcium condition is intriguing and begs the question whether phosphate starvation or stress response are somehow involved in calcium homeostasis processes in the cell. Phosphate limitation can subsequently induce $\operatorname{arc} A B^{22}$.

It is interesting to note that $\operatorname{arc} A$ is upregulated 5-fold in both $\mathrm{CalC}$ and CalD in elevated/depleted calcium conditions (Figures 5 and 6). In addition, $\operatorname{arc} A$ induction is evident in Tnpho $A$ mutants PhoC23 (2-fold) and Cal134 (5-fold) in elevated calcium (Figure 7) but not in depleted calcium condition (Figure 8). Furthermore, increased expression of rpoS (8-fold in CalC and 10-fold in CalD) in elevated calcium condition (Figure 5) also may be indicative of a stress response. However, such a trend is not seen in the transposon mutants as rpoS level virtually remains unchanged when compared to the parental CC118 (Figures 7 and 8). There is a marginal but distinct increase in rpoS expression level in wild-type MG1655 with elevated and depleted calcium conditions as shown in Figure (Figure 1).

\section{ftsZ Expression}

Transposon mutant Cal134 demonstrated 50-fold upregulation of ftsZ (essential cell division protein FtsZ) during depleted calcium condition (Figure 8). The cell division mutant was 10 to 20 times larger than the parent CC118 and DAPI staining indicated exhibited condensed chromosomes within the cell ${ }^{1}$.

$\mathrm{CalC}$ and CalD mutants had 2-fold higher expression of $f t s Z$ when grown in the presence of calcium (Figure 5), but addition of EGTA did not result in any changes between the parental KBT001 and the mutants (Figure 6). Expression of this cell division gene in MG1655 was downregulated 2-fold in both elevated and depleted calcium conditions (Figure 1).

Calcium's role in cell division has been studied to a brief extent. Cytoplasmic calcium levels increase during cell division as observed with electron probe microanalysis and x-ray mapping of $E$. coli ${ }^{23}$. Prokaryotic cell cycle events are thought to be regulated through a single $\mathrm{Ca}^{2+}$ flux. FtsZ assembly from a monomer to oligomer is a magnesium-dependent process, requires guanine nucleotides and involves GTP hydrolysis ${ }^{24}$. FtsZ has also been shown to have in vitro calcium-stimulated polymerization and GTPase activity ${ }^{25}$. In our cell division mutant Cal134, overexpression of $f t s Z$ can explain the cell division defect as evidenced under the microscope ${ }^{1}$.

\section{pqiB Expression}

Paraquat, a superoxide radical-generating agent, induces $p q i B$ gene and it is regulated by the soxRS locus ${ }^{26}$. Elevated (Figure 7) and depleted (Figure 8) calcium conditions upregulated pqiB 
in PhoC23 (10-fold) while Cal134 demonstrated approx. 40-fold increase in $f t s Z$ expression in the presence of elevated calcium (Figure 7). The possible role of calcium homeostasis in oxidative stress during growth in elevated extracellular calcium condition is interesting to note. In contrast to the mutants, the wild type MG1655 showed downregulation (3 - 5-fold) of $p q i B$ gene in both elevated and depleted calcium conditions (Figure 1).

\section{corA Expression}

The magnesium/nickel/cobalt transporter gene cor $A$ was upregulated 180-fold in Cal134 and 15-fold in PhoC23 during depleted calcium condition (Figure 8). These mutants also exhibited overexpression (approx. 12-fold for PhoC23 and 32fold for Cal134) when exposed to high calcium (Figure 7). Calcium-sensitive TnphoA mutants were also sensitive to other divalent cations ${ }^{1}$.

The calA mutation that confers sensitivity to calcium as described by Brey and Rosen $(1979)^{16}$ was in a strain that also harbored a mutation in the corA locus. The corA mutants are sensitive to calcium suggesting that corA may have a role in calcium access to the interior of the cell ${ }^{12}$. We would speculate the cytosolic free calcium level in the transposon mutant Cal134 to be significantly different from wild type parent or PhoC23. Depletion of calcium seems to have a drastic effect on Cal134 as manifested by the dramatic upregulation of corA.

\section{atoA Expression}

The qRT-PCR results exhibited a 10-fold and 4-fold upregulation of atoA under elevated calcium conditions in the mutants CalC and CalD, respectively (Figure 5).

Theodorou and Kyriakidis (2009) ${ }^{20}$ investigated the involvement of external $\mathrm{Ca}^{2+}$ on cPHB [complexed poly-(R)-3hydroxybutyrate] biosynthesis in E. coli. The synthesis of cPHB is regulated by the AtoS-AtoC two-component system that in turn regulates the expression of the atoDAEB operon. In their study, growth of $E$. coli in the presence of increased calcium $(0 \mathrm{mM}, 0.25 \mathrm{mM}, 0.50 \mathrm{mM}, 1.0 \mathrm{mM}, 5.0 \mathrm{mM})$ resulted in concentration-dependent induction of cPHB biosynthesis. Maximal cPHB levels accumulated at higher calcium concentrations (2.5mM). Addition of EGTA downregulated cPHB biosynthesis but EGTA-mediated down-regulation of cPHB biosynthesis was circumvented by the addition of calcium and magnesium ${ }^{20}$.

Our mutants CalC and CalD have cytoplasmic free calcium concentration of $1130 \mathrm{nM}$ and $410 \mathrm{nM}$, respectively ${ }^{7}$. During qRT-PCR experiments, CalC in elevated extracellular calcium condition exhibited 10-fold induction of atoA (Figure 5). Similarly, CalD with intracellular free calcium of $410 \mathrm{nM}$ exhibited 4-fold upregulation of atoA (Figure 5). Treatment with EGTA resulted in roughly 2-fold upregulation in these mutants (Figure 6).
Cytoplasmic $\mathrm{Ca}^{2+}$ levels are not regulated in the calC and calD mutants ${ }^{99}$. Growth of these mutants in the presence of EGTA had lower cytoplasmic $\mathrm{Ca}^{2+}$ levels than those cells grown in the absence of EGTA. The addition of calcium to the growth medium resulted in elevated levels of cytoplasmic $\mathrm{Ca}^{2+}$ levels for these two cal mutants. It will be very interesting to employ calC mutant (with cytoplasmic free calcium concentration of $1130 \mathrm{nM}$ ) to further characterize the involvement of calcium homeostasis during cPHB production. As PHB is a viable polymer of tremendous importance in industry, elucidating the correlation between calcium homeostasis and PHB synthesis may lead to construction of better PHB-producing strains.

\section{$y f a D$ and $y b b O$ Expression}

The expression of $y f a D$ that codes for a conserved protein (unknown function) was upregulated 35-40 fold in both elevated and depleted calcium conditions in transposon mutant PhoC23 (Figures 7 and 8). Cal134 exhibited approx. 38-fold upregulation under elevated calcium condition. However, $y f a D$ was repressed between 5 - 10-fold in MG1655 under elevated and depleted calcium conditions (Figure 1).

Another gene $y b b O$ (unknown function) was upregulated approx. 9-fold in CalC mutant and 4-fold in CalD mutant under elevated calcium conditions (Figure 5). These mutants exhibited almost 2-fold induction under depleted calcium conditions (Figure 6). While it is very likely that these genes are under calcium regulation as evidenced by changes in gene expression in elevated or depleted calcium, we have very little information available to assign any biological function quite yet.

\section{cheB Expression}

The mutants, calC and calD, have high levels of cytoplasmic calcium. Both of the cal mutants are also defective in chemotaxis ${ }^{99}$. During chemotaxis, chemoreceptors are controlled by methylation and demethylation. A specific protein methylesterase (methyl-accepting chemotaxis protein-glutamate methylesterase), the product of the cheB gene, catalyzes the demethylation reaction.

Our qRT-PCR results showed the gene $c h e B$ slightly upregulated in elevated calcium condition in both calC and calD mutants (Figure 5). However, in depleted calcium condition the gene was downregulated in both mutants (Figure 6).

Calcium's regulatory role in bacterial genome likely includes a great many diverse genes. While it is relatively non-remarkable to witness the cell division defect in calcium-sensitive mutants because of a priori knowledge of calcium involvement in FtsZ polymerization, it is quite intriguing to see calcium's involvement in stress response. Whether it is a direct or indirect regulatory process that culminates in the upregulation of $\operatorname{mar} B$ in all calciumsensitive mutants, or approx.10-fold induction of rpoS in elevated extracellular calcium condition in calC or calD mutants - many questions are raised regarding calcium's role in stress response when looking at the quantitative qRT-PCR data. A systematic 
approach that utilizes the mutants, incorporates accurate measurement of cytoplasmic free calcium, encompasses genomewide microarray studies with subsequent qRT-PCR validation of a more robust subset of genes is warranted. Also interesting is the fact that there is no calcium influx gene that is identified yet. A greater understanding of the role of calcium in PHB synthesis and perhaps calcium sequestration within the cell may shed light on more interesting details of calcium homeostasis in bacterial cells.

\section{Acknowledgements}

This investigation was supported in part by Grant-in-Aid 96077285 from The American Heart Association and by the College of Life Science and Agriculture, The University of New Hampshire.

\section{References:}

1. Smith RJ. 1995. Calcium and bacteria. Advances in Microbial Physiology. 37: 83-133.

2. Norris V, Grant S, Freestone P, Ganvin J, Sheikh FN, Toth I, Trinei M, Modha K and Norman RI. 1996. Calcium signalling in bacteria. $J$. Bacteriol. 178: $3677-3682$.

3. Hogarth B and Ellar CJ. 1978. Calcium accumulation during sporulation of Bacillus megaterium. Biochem. J. 176: 197-203.

4. Ordal GW. 1977. Calcium ion regulates chemotactic behaviour in bacteria. Nature (London). 270: 66-67.

5. Tisa KS and Adler J. 1992. Calcium ions are involved in Escherichia coli chemotaxis. Proc. Natl. Acad. Sci.USA. 89: 11804-11808.

6. Rasmussen H and Rasmussen J E. 1990. Calcium as intracellular messenger: from simplicity to complexity. Curr. Top. Cell. Regul. 31: 1109.

7. Tisa KS and Adler J. 1995a. Chemotactic properties of Escherichia coli mutants having abnormal $\mathrm{Ca}^{2+}$ content. J. Bacteriol. 177(24): 7112-7118.

8. Tisa KS and Adler J. 1995b. Cytoplasmic free- $\mathrm{Ca}^{2+}$ level rises with repellents and falls with attractants in Escherichia coli chemotaxis. Proc. Natl. Acad. Sci. USA. 92: 10777-10781. 102.

9. Tisa KS, Olivera B and Adler J. 1993. Inhibition of Escherichia coli chemotaxis by ?-conotoxin, a calcium channel blocker. J. Bacteriol. 175: 1235-1238. 103.

10. Tisa KS, Sekelsky JJ and Adler J. 2000. Effects of Organic Antagonists of $\mathrm{Ca} 2+, \mathrm{Na}+$, and $\mathrm{K}+$ on Chemotaxis and Motility of Escherichia coli. $J$. Bacteriol. 182: 4856-4861.

11. Watkins NJ, Knight MR, Trewavas AJ and Campbell AK. 1995. Free calcium transients in chemotactic and non-chemotactic strains of
Escherichia coli determined by using recombinant aequorin. Biochem. $J$. 306: $865-869$.

12. Gangola P and Rosen BP. 1987. Maintenance of intracellular calcium in Escherichia coli. J. Biol. Chem. 262: 12570-12574.

13. Naseem R, Wann KT, Holland IB and Campbell AK. 2009. ATP regulates calcium efflux and growth in E. coli. J. Mol. Biol. 391: 42-56.

14. Arif M, Howard J and Tisa LS. 2014. Calcium Homeostasis in Escherichia coli: Characterization of Mutants and Genome Expression of MG1655. Bangladesh Journal of Microbiology. 31(1-2): 1-8.

15. Manoil C and Beckwith J. 1985. TnphoA:a transposon probe for protein export signals. Proc. Natl. Acad. Sci. USA. 82: 8129-8133.

16. Brey RN and Rosen BP. 1979. Properties of Escherichia coli mutants altered in calcium/proton antiport activity. J. Bacteriol. 139: 824-834.

17. Blattner FR, Plumkett III G, Bloch BA, Perna NT, Burland V, Riley M, Collado-Vides J, Glasner JC, Rode BK, Mayhew GF, Gregor J, Davis NW, Krirkpatrick HA, Goeden MA, Rose CJ, Mau B and Shao Y. 1997. The complete genome sequence of Escherichia coli K-12. Science. 277: 1453-1474.

18. Gutierrez C, Barondess J, Manoil C and Beckwith J. 1987. The use of transposon TnphoA to detect genes for cell envelope proteins subject to a common regulatory stimulus: Analysis of osmotically regulated genes in Escherichia coli. Journal of Molecular Biology. 195(2): 289-297.

19. Pfaffl MW 2001. A new mathematical model for relative quantification in real-time RT-PCR Nucl. Acids Res. 29: 2002-2007.

20. Theodorou M, Tiligada E and Kyriakidis C. 2009. Extracellular $\mathrm{Ca}^{2+}$ transients affect poly-(R)-3-hydroxybutyrate regulation by the AtoS-A toC system in Escherichia coli. Biochem. J. 417: 667-672

21. Park MH, Wong BB, and Lusk JE. 1976. Mutants in three genes affecting transport of magnesium in Escherichia coli: genetics and physiology. $J$. Bacteriol. 126: 1096-1103.

22. Marzan KW and Shimizu K. 2011. Metabolic regulation of Escherichia coli and its phoB and phoR genes knockout mutants under phosphate and nitrogen limitations as well as at acidic condition. Microbial Cell Factories. 10: 39-54.

23. Chang B-F, Shuman H and Somlyo AP. 1986. Electron probe analysis, xray mapping, and electron energy-loss spectroscopy of calcium, magnesium, and monovalent ions in log-phase and in dividing Escherichia coli B cells. J. Bacteriol. 167: 935-939.

24. Mukherjee A, Dai K and Lutkenhaus J. 1992. Escherichia coli cell division protein FtsZ is a guanine nucleotide binding protein. Proc. Natl. Acad. Sci. USA. 90: 1053-1057.

25. Yu X-B and Margolin W. 1997. Ca2ÿÛÜ-mediated GTP-dependent assembly of bacterial cell division protein FtsZ into asters and polymer networks in vitro. EMBO J. 16: 5455-5463.

26. Koh YS and Roe JH. 1995. Isolation of a novel paraquat-inducible (pqi) gene regulated by the soxRS locus in Escherichia coli.J Bacteriol. 177(10): 2673-8 